TU-569

RCNS-99-04

AMES-HET-99-07

July 1999

\title{
$R$-parity violation and top quark polarization at the Fermilab Tevatron collider
}

\author{
Ken-ichi Hikasa and Jin Min Yang \\ Department of Physics, Tohoku University, Aoba-ku, Sendai 980-8578, Japan \\ Bing-Lin Young \\ Department of Physics and Astronomy, Iowa State University, Ames, Iowa 50011, USA
}

\begin{abstract}
The lepton or baryon number violating top quark interactions in the supersymmetric standard model with $R$ parity violation contribute to the process $d \bar{d} \rightarrow t \bar{t}$ at the tree level via the $t$ - or $u$-channel sfermion exchange. Since these interactions are chiral, they induce polarization to the top quark in the $t \bar{t}$ events at hadron colliders. We show in this article that the polarization can be a useful observable for probing these interactions at the upgraded Fermilab Tevatron collider, because the polarization is expected to be very small in the standard model.
\end{abstract}




\section{INTRODUCTION}

The top quark has an exceptionally large mass of the order of the electroweak symmetry breaking scale and is naturally expected to have a close connection to new physics [i]]. The Run 1 of the Fermilab Tevatron collider has successfully ended with the discovery of the top quark, but has yielded only a relatively small number of top quark events, which leaves plenty of room for new physics to be uncovered with the Tevatron upgrade [2] in the near future. Due to higher statistics, the $t \bar{t}$ sample at the upgraded Tevatron is expected to provide a sensitive probe of new physics 3, 3. 4.

A most popular model of new physics is the Minimal Supersymmetric Model (MSSM). In this model, the discrete multiplicative symmetry of $R$-parity, defined by $R=(-1)^{2 S+3 B+L}$ with spin $S$, baryon number $B$ and lepton number $L$, is often imposed on the Lagrangian to maintain the separate conservation of $B$ and $L$. This conservation requirement is, however, not dictated by any fundamental principle such as gauge invariance or renormalizability. The finiteness of the neutrino mass as suggested by the Super-Kamiokande and several other neutrino experiments [5] also implies that lepton numbers may be violated.]

The most general superpotential of the MSSM consistent with the $\mathrm{SU}(3) \times \mathrm{SU}(2) \times \mathrm{U}(1)$ symmetry and supersymmetry contains $R$-violating interactions which are given by [6]

$$
\mathcal{W}_{\not R}=\frac{1}{2} \lambda_{i j k} L_{i} L_{j} E_{k}^{c}+\lambda_{i j k}^{\prime} \delta^{\alpha \beta} L_{i} Q_{j \alpha} D_{k \beta}^{c}+\frac{1}{2} \lambda_{i j k}^{\prime \prime} \epsilon^{\alpha \beta \gamma} U_{i \alpha}^{c} D_{j \beta}^{c} D_{k \gamma}^{c}+\mu_{i} L_{i} H_{2} .
$$

Here $L_{i}\left(Q_{i}\right)$ and $E_{i}\left(U_{i}, D_{i}\right)$ are the left-handed lepton (quark) doublet and right-handed lepton (quark) singlet chiral superfields, and $c$ denotes charge conjugation. $H_{1,2}$ are the Higgs chiral superfields. The indices $i, j, k$ denote generations and $\alpha, \beta$ and $\gamma$ are the color indices. The $\lambda$ and $\lambda^{\prime}$ are the coupling constants of the $L$-violating interactions and $\lambda^{\prime \prime}$ those of the $B$-violating interactions. The non-observation (so far) of the proton decay imposes very strong constraints on the product of the $L$-violating and $B$-violating couplings [0]. It is thus conventionally assumed in phenomenological studies that only one type of these interactions (either $L$ - or $B$-violating) exists. Constraints on these $R$-parity violating couplings have been obtained from various analyses [8 14] and some of their phenomenological implications at lepton [15] and hadron [16] colliders have been investigated. It is notable that the bounds on the couplings involving top quark are generally quite weak (see Ref. [17] for a review). In the future precision top quark experiments at the upgraded Tevatron, these couplings may either manifest themselves or subjected to further, stronger constraints.

In proton-antiproton collisions, the $L$-violating top quark interaction $\lambda^{\prime}$ can induce treelevel processes such as $d \bar{d} \rightarrow t_{L} \bar{t}_{L}$ via slepton exchange in the $t$ channel. (Here the subscript $L$ stands for chirality, not helicity.) Similarly, the $B$-violating $\lambda^{\prime \prime}$ coupling gives rise to the process $d \bar{d} \rightarrow t_{R} \bar{t}_{R}$ via squark exchange. Since the couplings are chiral, these new production mechanisms produce an asymmetry between the left- and right-handed polarized top quarks. This polarization can be a sensitive probe for these couplings due to the fact that both the

\footnotetext{
${ }^{1}$ In fact, the $L$-violating interactions of the $R$-violating MSSM can give rise to neutrino masses at the one-loop level [6].
} 
SM and $R$-conserving MSSM contributions to the polarization are small[2].

In Ref. [19], the contribution of these processes to the total $t \bar{t}$ cross section was studied to constrain the relevant $R$-violating couplings. The effect is found to be at most of the level of a few percent. Because of the uncertainties in the SM predictions of the $t \bar{t}$ production cross section and possible contributions from the $R$-conserving MSSM interactions [20], the total $t \bar{t}$ cross section turns out not to be a suitable observable for the R-parity violating interactions. In this paper, we investigate the possibility of probing the $R$-violating in several observables including the top quark polarization in $t \bar{t}$ production at the upgraded Tevatron collider. The top polarization can serve as a sensitive probe of the R-parity violating interactions because of the negligibly small SM or MSSM contribution.

\section{PROBING $R$-VIOLATION VIA TOP POLARIZATION}

In terms of the four-component Dirac notation, the Lagrangian of the $\lambda^{\prime}$ and $\lambda^{\prime \prime}$ interactions is given by (notice that $\lambda_{i j k}^{\prime \prime}=-\lambda_{i k j}^{\prime \prime}$ )

$$
\begin{gathered}
\mathcal{L}_{\lambda^{\prime}}=-\lambda_{i j k}^{\prime}\left[\tilde{\nu}_{L}^{i} \bar{d}_{R \alpha}^{k} d_{L}^{j \alpha}+\tilde{d}_{L}^{j \alpha} \bar{d}_{R \alpha}^{k} \nu_{L}^{i}+\left(\tilde{d}_{R}^{k \alpha}\right)^{*}\left(\bar{\nu}_{L}^{i}\right)^{c} d_{L}^{j \alpha}\right. \\
\left.-\tilde{e}_{L}^{i} \bar{d}_{R \alpha}^{k} u_{L}^{j \alpha}-\tilde{u}_{L}^{j \alpha} \bar{d}_{R \alpha}^{k} e_{L}^{i}-\left(\tilde{d}_{R}^{k \alpha}\right)^{*}\left(\bar{e}_{L}^{i}\right)^{c} u_{L}^{j \alpha}\right]+ \text { h.c. }, \\
\mathcal{L}_{\lambda^{\prime \prime}}=-\lambda_{i j k}^{\prime \prime} \epsilon_{\alpha \beta \gamma}\left[\left(\tilde{d}_{R}^{k \gamma}\right)^{*} \bar{u}_{R}^{i \alpha}\left(d_{R}^{j \beta}\right)^{c}+\frac{1}{2}\left(\tilde{u}_{R}^{i \alpha}\right)^{*} \bar{d}_{R}^{j \beta}\left(d_{R}^{k \gamma}\right)^{c}\right]+\text { h.c. }
\end{gathered}
$$

The relevant Feynman diagrams for the process $d \bar{d} \rightarrow t \bar{t}$ via the $L$ - and $B$-violating couplings are shown in Fig. 1. It is evident that these amplitudes are proportional to $\left|\lambda_{i 31}^{\prime}\right|^{2}$ and $\left|\lambda_{31 i}^{\prime \prime}\right|^{2}$, respectively. The form of these amplitudes are discussed in some detail in the Appendix. Here we show the cross section at $|\lambda|^{2}$ (the interference term with the SM $O\left(\alpha_{s}^{2}\right)$ amplitude), $d \hat{\sigma}_{\lambda_{t} \lambda_{\bar{t}}}^{\text {new }} / d \cos \theta^{*}$ for each $t, \bar{t}$ helicity state $\lambda_{t, \bar{t}}= \pm$. For the $L$-violating couplings, we find

$$
\begin{aligned}
\frac{d \hat{\sigma}_{+-}^{\text {new }}}{d \cos \theta^{*}} & =-\frac{\alpha_{s}\left|\lambda_{i 31}^{\prime}\right|^{2} \beta}{72 \hat{s}} \frac{(1-\beta)\left(1+\cos \theta^{*}\right)^{2}}{1+2\left(m_{\tilde{e}^{i}}^{2}-m_{t}^{2}\right) / \hat{s}-\cos \theta^{*}} \\
\frac{d \hat{\sigma}_{++}^{\text {new }}}{d \cos \theta^{*}}=\frac{d \hat{\sigma}_{--}^{\text {new }}}{d \cos \theta^{*}} & =-\frac{\alpha_{s}\left|\lambda_{i 31}^{\prime}\right|^{2} \beta m_{t}^{2}}{18 \hat{s}^{2}} \frac{\sin ^{2} \theta^{*}}{1+2\left(m_{\tilde{e}^{i}}^{2}-m_{t}^{2}\right) / \hat{s}-\cos \theta^{*}} \\
\frac{d \hat{\sigma}_{-+}^{\text {new }}}{d \cos \theta^{*}} & =-\frac{\alpha_{s}\left|\lambda_{i 31}^{\prime}\right|^{2} \beta}{72 \hat{s}} \frac{(1+\beta)\left(1-\cos \theta^{*}\right)^{2}}{1+2\left(m_{\tilde{e}^{i}}^{2}-m_{t}^{2}\right) / \hat{s}-\cos \theta^{*}}
\end{aligned}
$$

where $\hat{s}$ is the $t \bar{t}$ c.m. energy squared, $\theta^{*}$ the scattering angle in the $t \bar{t}$ c.m. frame, and $\beta=\left(1-4 m_{t}^{2} / \hat{s}\right)^{1 / 2}$. A sum over the flavor index $i$ is implied.

Similar processes $s \bar{s} \rightarrow t \bar{t}$ and $b \bar{b} \rightarrow t \bar{t}$ occurs through the couplings $\lambda_{i 32}^{\prime}$ and $\lambda_{i 33}^{\prime}$, respectively. The amplitudes for these processes are given by the same formulas with obvious

\footnotetext{
${ }^{2}$ For $\tan \beta<1$ and light gluino, which is disfavored by the existing experimental data, the polarization induced by supersymmetric weak and strong interactions could be significant, however [18].
} 
substitution of the coupling. We will neglect these processes as the parton distributions of $s$ and $b$ quarks are small in a proton.

The corresponding expressions for the SM (QCD) lowest-order amplitude is

$$
\begin{aligned}
& \frac{d \hat{\sigma}_{+-}^{0}}{d \cos \theta^{*}}=\frac{d \hat{\sigma}_{-+}^{0}}{d \cos \theta^{*}}=\frac{\pi \alpha_{s}^{2} \beta}{18 \hat{s}}\left(1+\cos ^{2} \theta^{*}\right), \\
& \frac{d \hat{\sigma}_{++}^{0}}{d \cos \theta^{*}}=\frac{d \hat{\sigma}_{--}^{0}}{d \cos \theta^{*}}=\frac{2 \pi \alpha_{s}^{2} \beta m_{t}^{2}}{9 \hat{s}^{2}} \sin ^{2} \theta^{*}
\end{aligned}
$$

The sum of these two contributions give the cross section $d \hat{\sigma}_{\lambda_{t} \lambda_{\bar{t}}} / d \cos \theta^{*}$ up to order $|\lambda|^{2}$.

When only the polarization of the top quark is observed, we can sum over the $\bar{t}$ helicity

$$
\frac{d \hat{\sigma}_{\lambda_{t}}}{d \cos \theta^{*}}=\frac{d \hat{\sigma}_{\lambda_{t}+}}{d \cos \theta^{*}}+\frac{d \hat{\sigma}_{\lambda_{t}-}}{d \cos \theta^{*}}
$$

Integrating over the scattering angle, we obtain

$$
\begin{aligned}
\hat{\sigma}_{+}^{0}=\hat{\sigma}_{-}^{0}= & \frac{4 \pi \alpha_{s}^{2} \beta}{27 \hat{s}}\left(1+\frac{2 m_{t}^{2}}{\hat{s}}\right), \\
\hat{\sigma}_{+}^{\text {new }}+\hat{\sigma}_{-}^{\text {new }}= & \frac{\alpha_{s}\left|\lambda_{i 31}^{\prime}\right|^{2} \beta}{9 \hat{s}}\left\{-\frac{1}{2}+\frac{m_{\tilde{e}^{i}}^{2}-m_{t}^{2}}{\hat{s}}\right. \\
& \left.-\frac{1}{\beta}\left[\frac{m_{t}^{2}}{\hat{s}}+\frac{\left(m_{\tilde{e}^{i}}^{2}-m_{t}^{2}\right)^{2}}{\hat{s}^{2}}\right] \log \frac{(1+\beta)^{2}+4 m_{\tilde{e}^{i}}^{2} / \hat{s}}{(1-\beta)^{2}+4 m_{\tilde{e}^{i}}^{2} / \hat{s}}\right\} \\
\hat{\sigma}_{+}^{\text {new }}-\hat{\sigma}_{-}^{\text {new }}= & \frac{\alpha_{s}\left|\lambda_{i 31}^{\prime}\right|^{2}}{9 \hat{s}}\left\{\frac{1}{2}-\frac{m_{\tilde{e}^{i}}^{2}-m_{t}^{2}}{\hat{s}}\right. \\
& \left.-\frac{1}{\beta}\left[\frac{m_{t}^{2}}{\hat{s}}-\frac{\left(m_{\tilde{e}^{i}}^{2}-m_{t}^{2}\right)^{2}}{\hat{s}^{2}}\right] \log \frac{(1+\beta)^{2}+4 m_{\tilde{e}^{i}}^{2} / \hat{s}}{(1-\beta)^{2}+4 m_{\tilde{e}^{i}}^{2} / \hat{s}}\right\}
\end{aligned}
$$

We define several observables sensitive to the $R$-violating interactions. The (total) top polarization is given by

$$
P_{t}=\frac{\hat{\sigma}_{+}-\hat{\sigma}_{-}}{\hat{\sigma}_{+}+\hat{\sigma}_{-}} .
$$

The hemisphere top polarizations are obtained by restricting the top scattering angle in the $t \bar{t}$ c.m. frame to the forward or backward hemisphere. The forward top polarization is defined as

$$
P_{t}^{F}=\frac{\hat{\sigma}_{+}\left(\cos \theta^{*}>0\right)-\hat{\sigma}_{-}\left(\cos \theta^{*}>0\right)}{\hat{\sigma}_{+}\left(\cos \theta^{*}>0\right)+\hat{\sigma}_{-}\left(\cos \theta^{*}>0\right)},
$$

and the backward top polarization $P_{t}^{B}$ is similarly defined with $\cos \theta^{*}<0$. The forwardbackward asymmetry of the top quark is given by

$$
A_{\mathrm{FB}}^{t}=\frac{\hat{\sigma}\left(\cos \theta^{*}>0\right)-\hat{\sigma}\left(\cos \theta^{*}<0\right)}{\hat{\sigma}\left(\cos \theta^{*}>0\right)+\hat{\sigma}\left(\cos \theta^{*}<0\right)},
$$

which can be defined for either a fixed top quark helicity or with the helicities summed. 
In the case of the $B$-violating interaction, the helicity cross sections are obtained from those for the $L$-violating case with the replacement $\cos \theta^{*} \rightarrow-\cos \theta^{*}$ and $\lambda_{t, \bar{t}} \rightarrow-\lambda_{t, \bar{t}}$. We have

$$
\begin{gathered}
\frac{d \hat{\sigma}_{+-}^{\text {new }}}{d \cos \theta^{*}}=-\frac{\alpha_{s}\left|\lambda_{31 k}^{\prime \prime}\right|^{2} \beta}{72 \hat{s}} \frac{(1+\beta)\left(1+\cos \theta^{*}\right)^{2}}{1+2\left(m_{\tilde{d}^{k}}^{2}-m_{t}^{2}\right) / \hat{s}+\cos \theta^{*}}, \\
\frac{d \hat{\sigma}_{++}^{\text {new }}}{d \cos \theta^{*}}=\frac{d \hat{\sigma}_{--}^{\text {new }}}{d \cos \theta^{*}}=-\frac{\alpha_{s}\left|\lambda_{31 k}^{\prime \prime}\right|^{2} \beta m_{t}^{2}}{18 \hat{s}^{2}} \frac{\sin ^{2} \theta^{*}}{1+2\left(m_{\tilde{d}^{k}}^{2}-m_{t}^{2}\right) / \hat{s}+\cos \theta^{*}}, \\
\frac{d \hat{\sigma}_{-+}^{\text {new }}}{d \cos \theta^{*}}=-\frac{\alpha_{s}\left|\lambda_{31 k}^{\prime \prime}\right|^{2} \beta}{72 \hat{s}} \frac{(1-\beta)\left(1-\cos \theta^{*}\right)^{2}}{1+2\left(m_{\tilde{d}^{k}}^{2}-m_{t}^{2}\right) / \hat{s}+\cos \theta^{*}} .
\end{gathered}
$$

The expression for $P_{t}$ changes sign in addition to the replacement of the coupling and mass. The forward and backward hemispheres should be also exchanged.

The contribution of the electroweak process $q \bar{q} \rightarrow Z^{*} \rightarrow t \bar{t}$ is known to be small [18] and will be neglected in the following analysis. It is important to realize here that there is no interference with the QCD amplitude because of the orthogonal color structure. Thus the effect of the $R$-violating interaction can be sizable even for a comparable coupling strength.

We calculate the cross section $\sigma$ for $p \bar{p} \rightarrow t \bar{t}+X$ by convoluting the parton cross section $\hat{\sigma}$ discussed above with the parton distribution functions. We use the CTEQ3L parton distribution functions [21] with both the renormalization and factorization scales chosen to be $\mu=\hat{s}^{1 / 2}$. The top quark mass of $175 \mathrm{GeV}$ is used. We assume that the QCD correction factors (the $K$ factor) to $\sigma^{\text {new }}$ and $\sigma^{0}$ take the same value of 1.7 .

For the purpose of estimating the statistical sensitivity, we assume that only the leptonic modes $t \rightarrow W^{+} b \rightarrow \ell^{+} \nu_{\ell} b(\ell=e$ or $\mu)$ are used to identify the top quark. This is because charge measurement is needed for top/antitop identification and the reconstruction of the final state decay products is required for separating the top polarization states from decay angular distribution [22]. The statistical error for the top polarization is given by

$$
\delta P_{t}=\frac{\left[2\left(N_{+}^{2}+N_{-}^{2}\right)\right]^{1 / 2}}{\left(N_{+}+N_{-}\right)^{3 / 2}} \simeq \frac{1}{\sqrt{N_{+}+N_{-}}}
$$

where $N_{+}$and $N_{-}$are the numbers of right-handed and left-handed top events.

The polarization of $\bar{t}$ can also be used to increase the statistical sensitivity of the data. The total polarizations $P_{\bar{t}}$ and $P_{t}$ have the same magnitude but opposite in sign within our approximation, and the hemisphere $\bar{t}$ polarization can be calculated from the expressions given above. To be conservative, we do not use the $\bar{t}$ polarization in the following analyses.

We assume for simplicity that only one of the couplings dominates. For the $L$-violating couplings, $\lambda_{131}^{\prime}$ and $\lambda_{231}^{\prime}$ are already strongly constrained by atomic parity violation and $\nu_{\mu}$ deep-inelastic scattering [10], respectively. We may thus interpret our result as that on $\lambda_{331}^{\prime}$, in which case the scalar-tau is exchanged in the process. In the case of $B$-violating interactions, none of the relevant couplings have been well constrained by other processes.

In Fig. 2, we show the polarizations $P_{t}, P_{t}^{F}$, and $P_{t}^{B}$ normalized to $\left|\lambda_{331}^{\prime}\right|^{2}$ versus the scalar-tau mass. The figures can be also read as $-P_{t} /\left|\lambda_{31 i}^{\prime \prime}\right|^{2}$ versus the squark mass with 'forward' and 'backward' interchanged. If the coupling is of the order of the weak $\mathrm{SU}(2)$ 
gauge coupling, a polarization of several \% is expected, without contradicting with existing limits. The total polarization is smaller than hemisphere polarizations because of the cancellation between the two hemispheres. For the coupling $\lambda_{331}^{\prime}\left(\lambda_{31 i}^{\prime \prime}\right)$, the backward (forward) polarization has the largest magnitude of the three. In spite of the smaller number of events, the statistical sensitivity of the backward (forward) polarization is higher than that of the total polarization, as may be seen from Fig. 3. Because of the small electroweak contribution (much less than one per cent), the top polarization can be a useful probe of the $R$-violating couplings.

The forward-backward asymmetries for the top quark (for individual helicities and their sum) are shown in Fig. 4. The size of the effect is similar to the polarizations. The total asymmetry is smaller than the asymmetries of each helicity top events.

The $2 \sigma$ limit for $\lambda_{331}^{\prime}\left(\lambda_{31 i}^{\prime \prime}\right)$, which is defined by Eq. (2.13) with $P_{t}^{B} / \delta P_{t}^{B}=2\left(P_{t}^{F} / \delta P_{t}^{F}=\right.$ 2 ), versus scalar-tau (squark) mass is shown in Fig. 5 for the integrated luminosities of 2 $\mathrm{fb}^{-1}$ (Run 2) and $30 \mathrm{fb}^{-1}$ (Run 3). The current $2 \sigma$ limits from $Z$ decay at LEP-I [11] are also shown for comparison.

Note that the limits on $\lambda_{331}^{\prime}$ obtained from the decay $Z \rightarrow \tau^{+} \tau^{-}$depend on squark mass since the coupling $\lambda_{331}^{\prime}$ contributes to this decay via the squark-quark loops. But our limits depend on the scalar-tau mass. Therefore, $P_{t}$ and $R_{\tau}$ can provide complementary information for the coupling $\lambda_{331}^{\prime}$, and the upgraded $2 \mathrm{TeV}$ Tevatron collider with $L=30 \mathrm{fb}^{-1}$ or even lower luminosity can provide meaningful information on this kind of new physics. As the polarization grows with energy, one can probe further parameter space in the couplings and masses at higher energies.

\section{SUMMARY}

We have studied the possibility to probe the $R$-parity violating interactions of the top quark at the Tevatron collider. Obviously, $R$-violating decays of squarks provide a way to limit these interactions [23]. We have shown that even when the squark is rather heavy and the production cross section is low, the $R$-violating interactions can affect the top quark pair production cross section via slepton (or squark) exchange. As these couplings are chiral, their contributions can lead to sizable polarization and forward-backward asymmetry for the top quark, that exceed the level expected from the standard model electroweak interactions. Hence, we expect that the forthcoming new runs at the Tevatron collider with main injector can improve the constraints on those couplings.

\section{ACKNOWLEDGMENTS}

JMY acknowledges JSPS for financial support. This work is supported in part by the Grant-in-Aid for Scientific Research (No. 10640243) and Grant-in-Aid for JSPS Fellows (No. 97317) from the Japan Ministry of Education, Science, Sports, and Culture. BLY acknowledges the support by a NATO collaborative grant. BLY would also like to thank

\footnotetext{
${ }^{3}$ Here we neglect the possible systematic error and only consider the statistical error.
} 
Professor Zhongyuan Zhu and Professor Ting-Kuo Lee, respectively at the Institute of Theoretical Physics, Chinese Academy of Sciences, Beijing and the National Center of Theoretical Sciences, Hsin Chu, for their kind hospitalities and support.

\section{APPENDIX}

Here we list the amplitudes for $d \bar{d} \rightarrow t \bar{t}$ used in the paper. The Feynman amplitudes are as follows:

$s$-channel gluon exchange

$$
\mathcal{M}_{s}=\frac{g_{s}^{2}}{s} \bar{u}(p) \gamma^{\mu} v(\bar{p}) \bar{v}(\bar{k}) \gamma_{\mu} u(k)\left(T^{a}\right)_{\gamma \delta}\left(T^{a}\right)_{\beta \alpha}
$$

$t$-channel slepton exchange

$$
\mathcal{M}_{t}=\frac{\left|\lambda^{\prime}\right|^{2}}{t-m_{\tilde{\ell}}^{2}} \bar{u}(p) \frac{1+\gamma_{5}}{2} u(k) \bar{v}(\bar{k}) \frac{1-\gamma_{5}}{2} v(\bar{p}) \delta_{\gamma \alpha} \delta_{\delta \beta}
$$

$u$-channel squark exchange

$$
\mathcal{M}_{u}=\frac{\left|\lambda^{\prime \prime}\right|^{2}}{u-m_{\tilde{q}}^{2}} \bar{u}(p) \frac{1-\gamma_{5}}{2} C \bar{v}^{T}(\bar{k}) u^{T}(k) C^{\dagger} \frac{1+\gamma_{5}}{2} v(\bar{p}) \epsilon_{\delta \alpha \epsilon} \epsilon_{\gamma \beta \epsilon}
$$

In these formulas, $\lambda^{\prime}$ and $\lambda^{\prime \prime}$ are to be interpreted as $\lambda_{i 31}^{\prime}$ and $\lambda_{31 k}^{\prime \prime}$.

After Fierz rearrangement, crossed channel amplitudes can be written in the $s$-channel form

$$
\begin{aligned}
\mathcal{M}_{t} & =\frac{\left|\lambda^{\prime}\right|^{2}}{t-m_{\tilde{\ell}}^{2}} \bar{u}(p) \gamma^{\mu} \frac{1-\gamma_{5}}{2} v(\bar{p}) \bar{v}(\bar{k}) \gamma_{\mu} \frac{1+\gamma_{5}}{2} u(k) \delta_{\gamma \alpha} \delta_{\delta \beta} \\
\mathcal{M}_{u} & =-\frac{\left|\lambda^{\prime \prime}\right|^{2}}{u-m_{\tilde{q}}^{2}} \bar{u}(p) \gamma^{\mu} \frac{1+\gamma_{5}}{2} v(\bar{p}) \bar{v}(\bar{k}) \gamma_{\mu} \frac{1+\gamma_{5}}{2} u(k) \delta_{\gamma \alpha} \delta_{\delta \beta}
\end{aligned}
$$

The helicity amplitudes for the process may be calculated from these invariant amplitudes. The amplitude for the helicity combination

$$
d\left(\lambda_{d}\right)+\bar{d}\left(\bar{\lambda}_{d}\right) \rightarrow t\left(\lambda_{t}\right)+\bar{t}\left(\bar{\lambda}_{t}\right)
$$

has the structure

$$
\mathcal{M}=\widetilde{\mathcal{M}}\left(E_{\mathrm{cm}}, \cos \theta\right) d_{\lambda_{i} \lambda_{f}}^{1}(\theta) e^{i\left(\lambda_{i}-\lambda_{f}\right) \varphi}
$$

where $\lambda_{i}=\lambda_{d}-\bar{\lambda}_{d}, \lambda_{f}=\lambda_{t}-\bar{\lambda}_{t},(\theta, \varphi)$ the scattering angle (the direction of the final $t$ with respect to the initial $d$ ), and $d_{\lambda \lambda^{\prime}}^{1}$ is the Wigner $d$ function (rotation matrix) for the spin-1 representation.

Since chirality is conserved, the process occurs for only two of the initial states with $\lambda_{i}= \pm 1$. The standard model contribution is 


$$
\widetilde{\mathcal{M}}_{s}= \begin{cases}-g_{s}^{2} C_{s} & \lambda_{f}= \pm 1 \\ -\frac{1}{\sqrt{2} \gamma} g_{s}^{2} C_{s} & \lambda_{f}=0\end{cases}
$$

The slepton exchange contribution exists only for $\lambda_{i}=+1$ because of the chirality structure of the interaction (only $d_{L}$ interacts):

$$
\widetilde{\mathcal{M}}_{t}= \begin{cases}-\left|\lambda^{\prime}\right|^{2} \frac{s}{2\left(t-m_{\tilde{e}}^{2}\right)}(1 \mp \beta) C_{t} & \lambda_{f}= \pm 1 \\ -\left|\lambda^{\prime}\right|^{2} \frac{s}{2\left(t-m_{\tilde{e}}^{2}\right)} \times \frac{1}{\sqrt{2} \gamma} C_{t} & \lambda_{f}=0\end{cases}
$$

The squark exchange contribution is also for $\lambda_{i}=+1$ only:

$$
\widetilde{\mathcal{M}}_{u}= \begin{cases}-\left|\lambda^{\prime \prime}\right|^{2} \frac{s}{2\left(u-m_{\tilde{d}}^{2}\right)}(1 \pm \beta) C_{u} & \lambda_{f}= \pm 1 \\ -\left|\lambda^{\prime \prime}\right|^{2} \frac{s}{2\left(u-m_{\tilde{d}}^{2}\right)} \times \frac{1}{\sqrt{2} \gamma} C_{u} & \lambda_{f}=0\end{cases}
$$

where $\beta=\left(1-4 m_{t}^{2} / s\right)^{1 / 2}$ is the top c.m. velocity, $\gamma=\left(1-\beta^{2}\right)^{-1 / 2}=\sqrt{s} / 2 m_{t}$, and the color factors are given by

$$
\begin{aligned}
C_{s} & =\delta_{\alpha \gamma} \delta_{\beta \delta}-\frac{1}{3} \delta_{\alpha \beta} \delta_{\gamma \delta} \\
C_{t} & =\delta_{\alpha \gamma} \delta_{\beta \delta} \\
C_{u} & =\delta_{\alpha \gamma} \delta_{\beta \delta}-\delta_{\alpha \beta} \delta_{\gamma \delta}
\end{aligned}
$$




\section{REFERENCES}

[1] For some of the general description of new physics in top quark sector, see: R. D. Peccei and X. Zhang, Nucl. Phys. B337, 269 (1990); R. D. Peccei, S. Peris and X. Zhang, Nucl. Phys. B349, 305 (1991); G. J. Gounaris, F. M. Renard, and C. Verzegnassi, Phys. Rev. D 52, 451 (1995); K. Whisnant, J. M. Yang, B.-L. Young and X. Zhang, Phys. Rev. D 56, 467 (1997); J. M. Yang and B.-L. Young, Phys. Rev. D 56, 5907 (1997); G. J. Gounaris, D. T. Papadamou, and F. M. Renard, Z. Phys. C 76, 333 (1997).

[2] Report of the tev_2000 Study Group, edited by D. Amidei and C. Brock, Fermilab-Pub96/082; A. P. Heinson, in QCD and High-Energy Hadronic Interactions, Proceedings of the XXXIst Rencontre de Moriond, Les Arcs, France, 1996, edited by Tran Thanh Van (Edition Frontiere, Gif-sur-Yvette, France, 1996), p. 43 (hep-ex/9605010).

[3] For some of the systematic studies of probing new physics from $t \bar{t}$ events at Tevatron, see: C. T. Hill and S. J. Parke, Phys. Rev. D 49, 4454 (1994); K. Hikasa, K. Whisnant, J. M. Yang and B.-L. Young, Phys. Rev. D 58, 114003 (1998).

[4] For some of the studies of probing anomalous top quark dipole moments in $t \bar{t}$ production at Tevatron, see: D. Atwood, A. Aeppli and A. Soni, Phys. Rev. Lett. 69, 2754 (1992); D. Atwood, A. Kagan, T. G. Rizzo, Phys. Rev. D 52, 6264 (1995); K. Cheung, Phys. Rev. D 53, 3604 (1996); P. Haberl, O. Nachtmann and A. Wilch, Phys. Rev. D 53, 4875 (1996); T. G. Rizzo, Phys. Rev. D 53, 6218 (1996); D. Silverman, Phys. Rev. D 54, 5562 (1996).

[5] Super Kamiokande Collaboration, Y. Fukuda et al., Phys. Rev. Lett. 81, 1562 (1998); T. Kajita, talk at Neutrino '98, Takayama, Japan, June, 1998; For a recent review of all relevant data on neutrion oscillations, see J.M. Conrad, summary talk at ICHEP '98, hep-ex/981109.

[6] For some of the earliest references on the phenomenology of R-violating supersymmetry, see: L. Hall and M. Suzuki, Nucl. Phys. B231, 419 (1984); J. Ellis et al., Phys. Lett. B 150, 142 (1985); G. Ross and J. Valle, Phys. Lett. B 151, 375 (1985); S. Dawson, Nucl. Phys. B261, 297 (1985); R. Barbieri and A. Masiero, Nucl. Phys. B267, 679 (1986).

[7] See, e.g., C. Carlson, P. Roy and M. Sher, Phys. Lett. B 357, 99 (1995); A. Y. Smirnov and F. Vissani, Phys. Lett. B 380, 317 (1996).

[8] B. Brahmachari and P. Roy, Phys. Rev. D 50, 39 (1994); J. L. Goity and M. Sher, Phys. Lett. B 346, 69 (1995).

[9] S. Dimopoulos and L. J. Hall, Phys. Lett. B 207, 210 (1987); R. M. Godbole, P. Roy and X. Tata, Nucl. Phys. B401, 67 (1993).

[10] V. Barger, G. F. Giudice and T. Han, Phys. Rev. D 40, 2978 (1989).

[11] J. M. Yang, hep-ph/9905486. For earlier studies, see G. Bhattacharyya, J. Ellis and K. Sridhar, Mod. Phys. Lett. A 10, 1583 (1995); G. Bhattacharyya, D. Choudhury and K. Sridhar, Phys. Lett. B 355, 193 (1995).

[12] K. Agashe and M. Graesser, Phys. Rev. D 54, 4445 (1996);

[13] D. Choudhury and P. Roy, Phys. Lett. B 378, 153 (1996).

[14] F. Zwirner, Phys. Lett. B 132, 103 (1983); R. N. Mohapatra, Phys. Rev. D 34, 3457 (1986); M. Hirsch, H. Kleingrothaus and S. G. Kovalenko, Phys. Rev. Lett. 75, 17 (1995); K. S. Babu and R. N. Mohapatra, Phys. Rev. Lett. 75, 2276 (1995); G. Bhattacharyya and D. Choudhury, Mod. Phys. Lett. A 10, 1699 (1995); D. E. Kaplan, hep-ph/9703347; J. Jang, J. K. Kim and J. S. Lee, Phys. Rev. D 55, 7296 (1997); 
G. Bhattacharyya and A. Raychaudhuri,Phys. Rev. D 57, 3837 (1998); J. M. Yang, B.L. Young and X. Zhang, Phys. Rev. D 58, 055001 (1998); T.-F. Feng, hep-ph/9806505. K. Huitu, K. Puolamaki, and D.-X. Zhang, Phys. Lett. B 446, 285 (1999).

[15] J. Erler, J. L. Feng and N. Polonsky, Phys. Rev. Lett. 78, 3063 (1997); J. L. Feng, J. F. Gunion and T. Han, Phys. Rev. D 58, 071701 (1998).

[16] A. Datta, J. M. Yang, B.-L. Young and X. Zhang, Phys. Rev. D 56, 3107 (1997); R. J. Oakes, K. Whisnant, J. M. Yang, B.-L. Young and X. Zhang, Phys. Rev. D 57, 534 (1998).

[17] G. Bhattacharyya, hep-ph/9709395; H. Dreiner, hep-ph/9707435.

[18] C. Kao, G. A. Ladinsky and C.-P. Yuan, FSU-HEP-930508, 1993 (unpublished); in Proceedings of DPF94, Albuquerque, NM, USA, 1994, edited by S. Seidel (World Scientific, Singapore), pp. 713-716; C. Kao, Phys. Lett. B 348, 155 (1995); C. S. Li, R. Oakes, J. M. Yang and C.-P. Yuan, Phys. Lett. B 398, 298 (1997); C. S. Li, C.-P. Yuan and H.-Y. Zhou, Phys. Lett. B 424, 76 (1998).

[19] D. K. Ghosh, S. Raychaudhuri and K. Sridhar, Phys. Lett. B 396, 177 (1997).

[20] C. S. Li, B. Q. Hu, J. M. Yang and C. G. Hu, Phys. Rev. D 52, 5014 (1995); J. Kim, J. L. Lopez, D. V. Nanopoulos and R. Rangarajan, Phys. Rev. D 54, 4364 (1996); J. M. Yang and C. S. Li, Phys. Rev. D 52, 1541 (1995); 54, 4380 (1996); C. S. Li, et al., Phys. Lett. B 379, 135 (1996); S. Alam, K. Hagiwara and S. Matsumoto, Phys. Rev. D 55, 1307 (1997); Z. Sullivan, Phys. Rev. D 56, 451 (1997); W. Hollik, W. M. Mosle and D. Wackeroth, Nucl. Phys. B516, 29 (1998).

[21] H. L. Lai et al., Phys. Rev. D 51, 4763 (1995).

[22] G. L. Kane, G. A. Ladinsky and C.-P. Yuan, Phys. Rev. D 45, 124 (1992); M. Jezabek and J. H. Kühn, Phys. Lett. B 329, 317 (1994); S. Parke and Y. Shadmi, Phys. Lett. B 387, 199 (1996).

[23] B. Allanach et al, "Searching for $R$-parity violation at Run-II of the Tevatron," edited by H. Dreiner, hep-ph/9906224. 


\section{FIGURES}

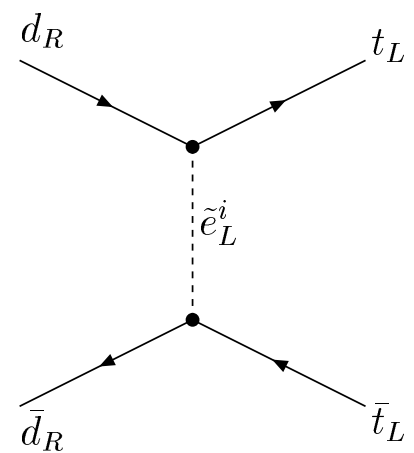

(a)

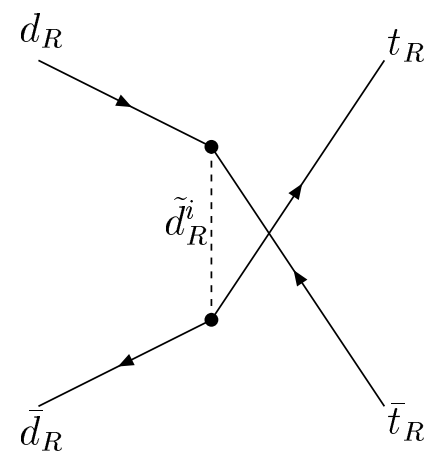

(b)

FIG. 1. Feynman diagrams for (a) the $L$-violating process $d \bar{d} \rightarrow t_{L} \bar{t}_{L}$ induced by $\lambda_{i 31}^{\prime}$, and (b) the $B$-violating process $d \bar{d} \rightarrow t_{R} \bar{t}_{R}$ induced by $\lambda_{31 i}^{\prime \prime}$. 


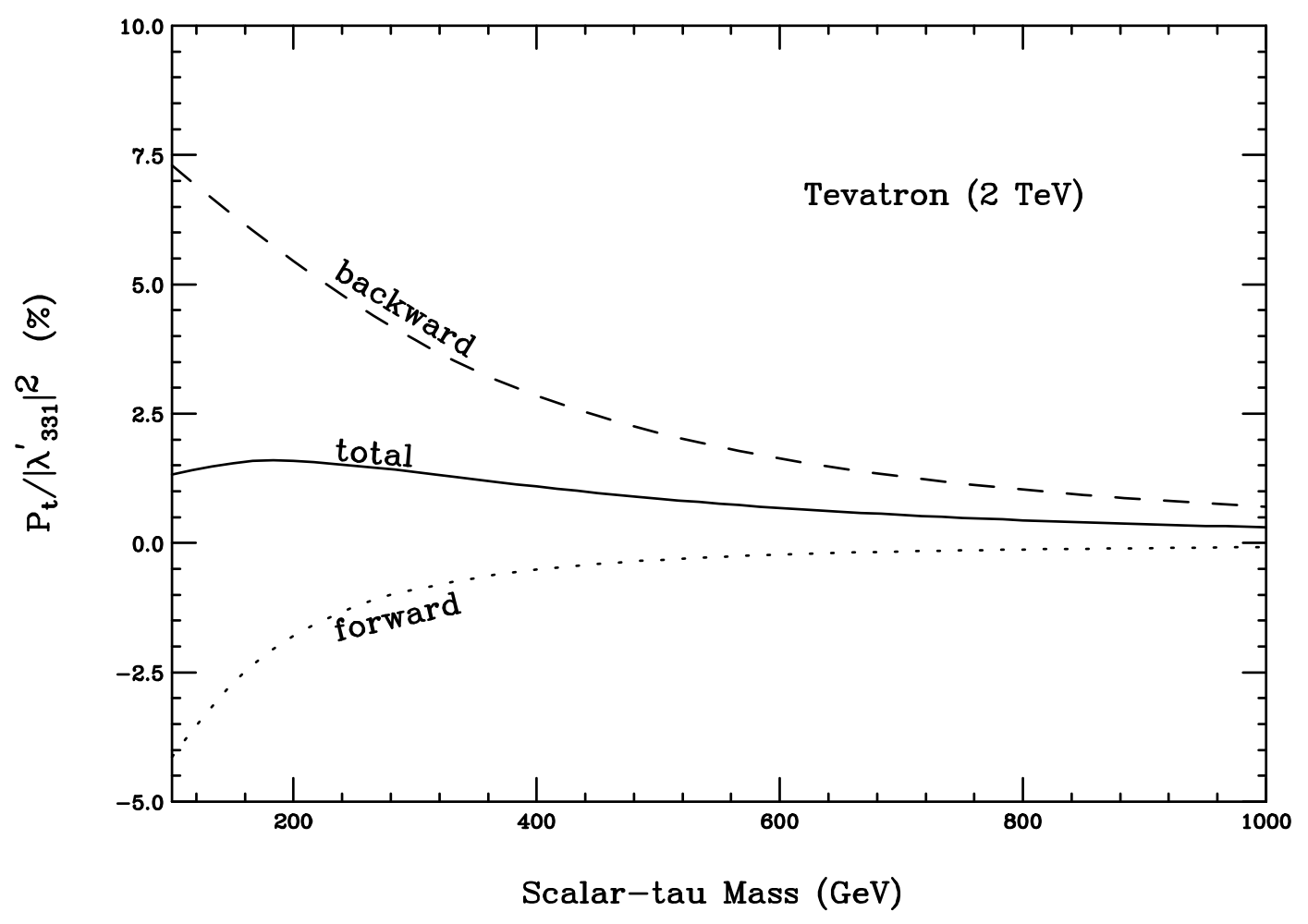

FIG. 2. Top quark polarizations at $\sqrt{s}=2 \mathrm{TeV}$ versus the exchanged scalar-tau mass. Total: $P_{t}$; forward: $P_{t}^{F}$ : backward: $P_{t}^{B}$. The figure can be also read as $-P_{t} /\left|\lambda_{31 i}^{\prime \prime}\right|^{2}$ versus the exchanged squark mass with 'forward' and 'backward' interchanged. 


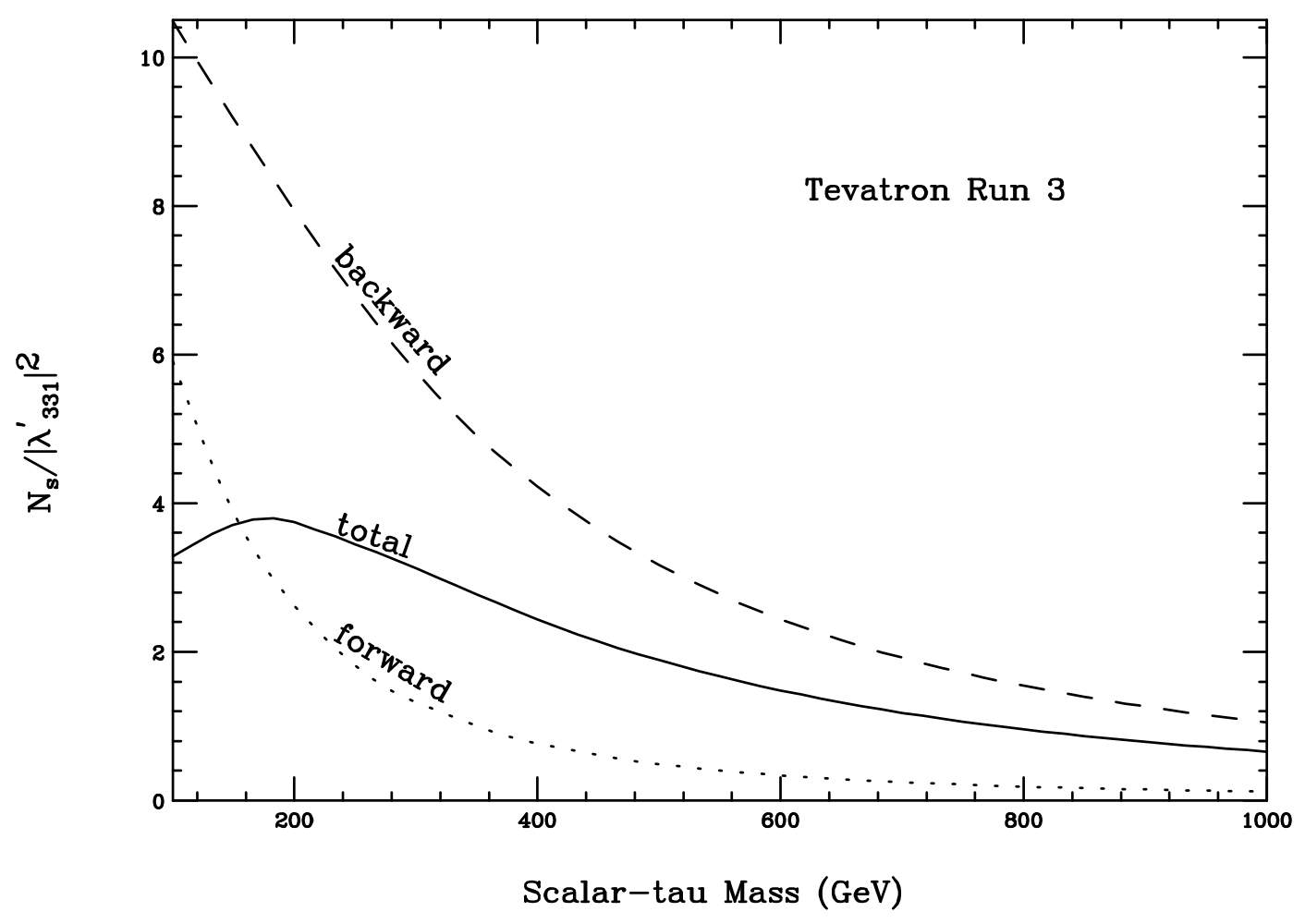

FIG. 3. Statistical significance at $\sqrt{s}=2 \mathrm{TeV}$ with $L=30 \mathrm{fb}^{-1}$. The figure can be also read as $N_{s} /\left|\lambda_{31 i}^{\prime \prime}\right|^{2}$ versus the exchanged squark mass with 'forward' and 'backward' interchanged. 


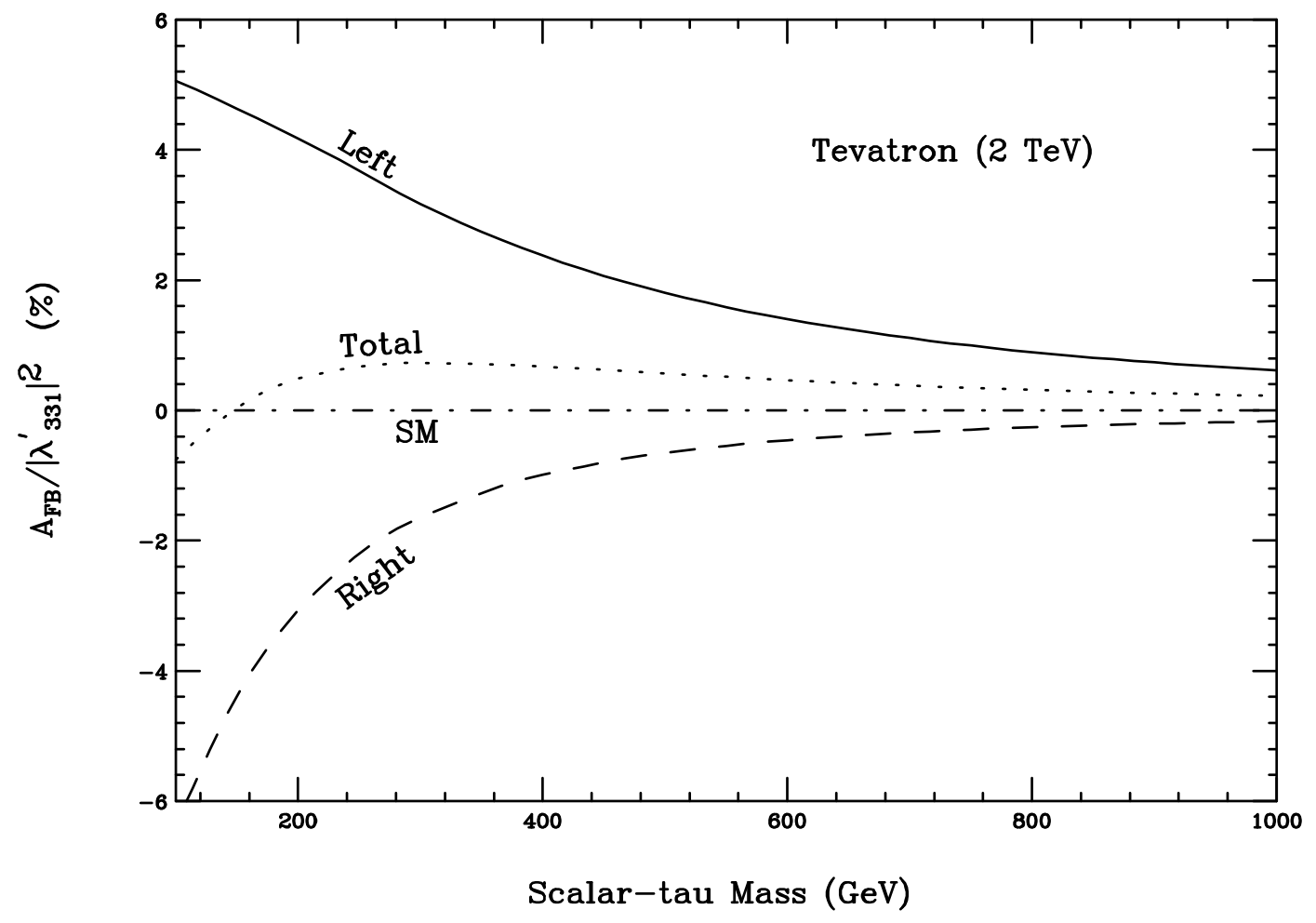

FIG. 4. Top quark forward-backward asymmetry at $\sqrt{s}=2 \mathrm{TeV}$ versus the exchanged sfermion mass for all events (total); left-handed (left) and right-handed (right) top events. The figure can be also read as $-A_{F B} /\left|\lambda_{31 i}^{\prime \prime}\right|^{2}$ versus the exchanged squark mass with 'Left' and 'Right' interchanged. 


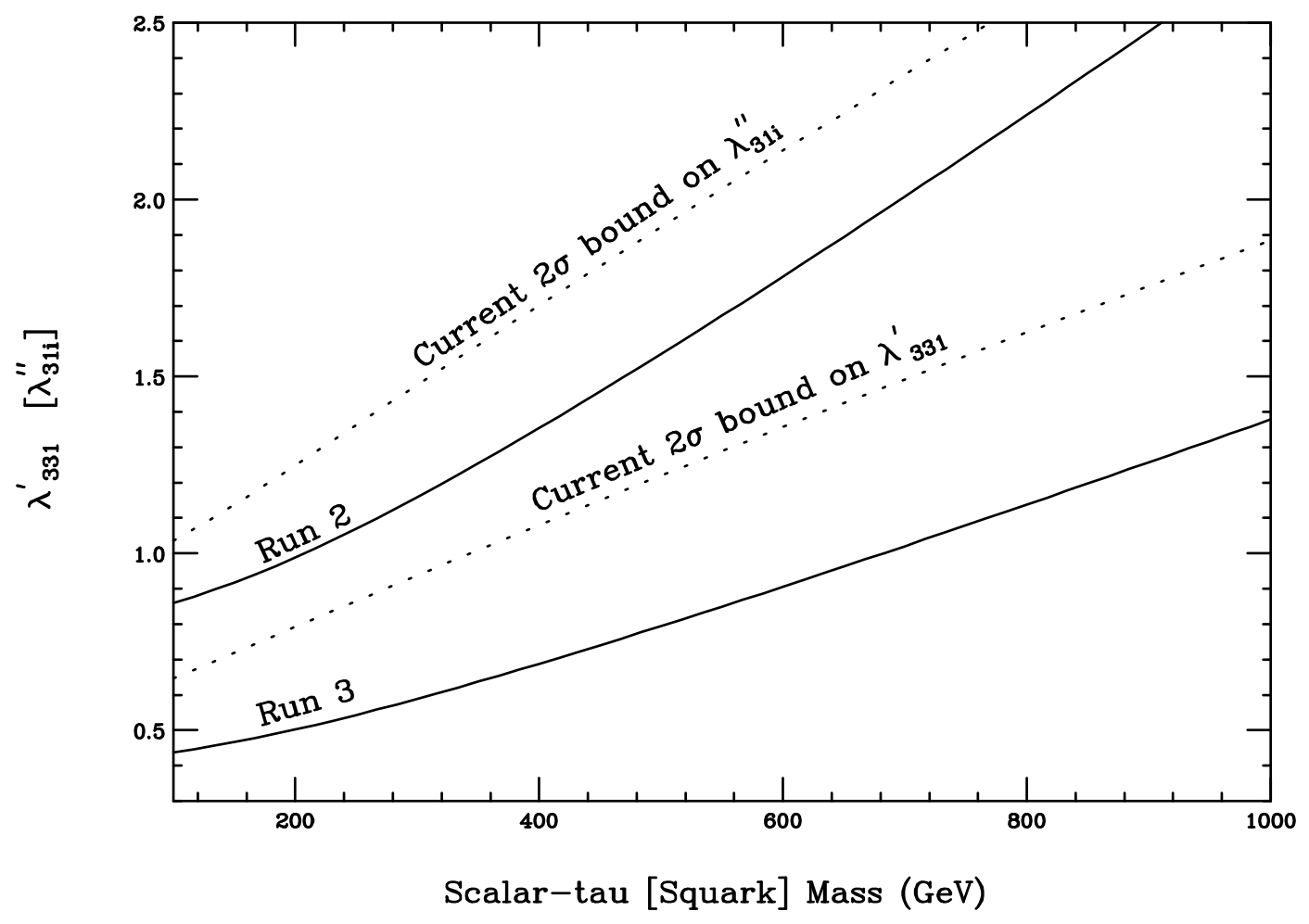

FIG. 5. The expected $2 \sigma$ limit for $\lambda_{331}^{\prime}\left(\lambda_{31 i}^{\prime \prime}\right)$ versus scalar-tau (squark) mass. The two solid curves are at $\sqrt{s}=2 \mathrm{TeV}$ with $L=2 \mathrm{fb}^{-1}$ (Run 2) and $30 \mathrm{fb}^{-1}$ (Run 3), respectively. The current limits from $Z$ decay are also shown as a function of the squark mass. 